BULLETIN OF THE

AMERICAN MATHEMATICAL SOCIETY

Volume 81, Number 1, January 1975

\title{
CONICAL DISTRIBUTIONS FOR RANK ONE SYMMETRIC SPACES
}

\author{
BY MEN-CHANG HU ${ }^{1}$
}

Communicated by S. S. Chern, June 10, 1974

Let $X=G / K$ be a symmetric space of noncompact type, where $G$ is a connected semisimple Lie group with finite center, and $K$ is the compact part of an Iwasawa decomposition $G=K A N$ of $G$. Let $M\left(M^{\prime}\right)$ be the centralizer (normalizer) of $A$ in $K$. Then the space $\Xi$ of all horocycles of $X$ can be identified with $G / M N$ or $(K / M) \times A[1, \mathrm{p} .8]$. The set of all smooth functions with compact supports on $\Xi$ endowed with the customary topology is denoted by $D(\Xi)$. Its dual $D^{\prime}(\Xi)$ consists of all distributions on $\Xi$. Let $W$ be the Weyl group $M^{\prime} / M$ and $\mathfrak{A}_{C}^{*}$ be the complex dual of $\mathfrak{A}$, the Lie algebra of $A$.

Definition [1, p. 65]. A distribution $\Psi \in D^{\prime}(\Xi)$ is said to be conical if (i) $\Psi$ is $M N$-invariant, (ii) $\Psi$ is an eigendistribution of every $G$-invariant differential operator on $\Xi$.

As is readily seen, this definition is parallel to that of spherical functions on $X$. On this basis $\mathrm{S}$. Helgason made the conjecture that the set of all conical distributions can be parametrized by $W \times \mathfrak{\Re}_{C}^{*}$, and he also established it in various cases [1, Chapter III, §4]. Our purpose here is to complete its verification in case $X$ has rank one.

Now for each $a \in A$, there is a map $\sigma(a)$ of $\Xi$ defined by $\sigma(a)(g M N)$ $=g a M N$. This gives rise to a corresponding action $\Psi \mapsto \Psi^{\sigma(a)}$ on the space $D^{\prime}(\Xi)$. If $\lambda \in \mathscr{A}_{C}^{*}$, let $D_{\lambda}^{\prime}=\left\{\Psi \in D^{\prime}(\Xi) \mid \Psi^{\sigma(a)}=e^{-(i \lambda+\rho) \log a} \Psi\right.$, $\forall a \in A\}$, where $\rho$ is half the sum of all positive restricted roots, counting multiplicity, and $\log : A \rightarrow \mathscr{U}$ is the inverse of the exponential map. The space $D_{\lambda}^{\prime}$ consists of the joint eigenspaces of the $G$-invariant differential operators on $\Xi\left[1\right.$, p. 69]. So an element $\Psi \in D^{\prime}(\Xi)$ is conical iff it is (i) $M N$-invariant, and (ii) belongs to some $D_{\lambda}^{\prime}$. Next we recall some constructions from [1, Chapter III, §4]. For each $s \in M^{\prime} / M$, choose an $m_{s} \in M^{\prime}$ in the

AMS (MOS) subject classifications (1970). Primary 43A85.

1 The author wishes to thank his thesis adivisor, Professor S. Helgason, for his guidance throughout this work. 
coset $s$ and put $\xi_{s}=m_{s} M N, \Xi_{s}=M N A \xi_{s}$. The group $M N A$ induces a regular Borel measure $d \nu_{s}$ on $\Xi_{s}$ which is invariant under $M N$ and $\sigma(a)$, $a \in A$. For $\xi \in \Xi_{s}$, let $a(\xi)$ be the unique element in $A$ such that $\xi \in$ $M N a(\xi) \xi_{s}$. Let $\langle$,$\rangle be the Killing form, \Sigma^{+}$the set of all positive restricted roots, $\Sigma^{-}=-\Sigma^{+}$, and $\Sigma_{0}^{+}$, the set of all indivisible roots in $\Sigma^{+}$.

THEOREM 1 [1,p. 82]. If $\lambda \in \mathfrak{A}_{C}^{*}$ satisfies $\operatorname{Re}\langle\alpha, i \lambda\rangle>0$ for all $\alpha$ in $\Sigma^{+} \cap s^{-1} \Sigma$, then the linear functional

$$
\Psi_{\lambda, s}^{\prime}: \phi \mapsto \int_{\Xi} \phi(\xi) \exp [(i s \lambda+s \rho)(\log a(\xi))] d \nu_{s}, \quad \phi \in O(\Xi)
$$

is a conical distribution in $D_{\lambda}^{\prime}$.

THEOREM $2[1$, p. 88]. Let

$$
d_{s}(\lambda)=\prod_{\alpha \in \Sigma_{0}^{+} \cap s^{-1} \Sigma_{0}^{-}} \Gamma\left(\frac{\langle i \lambda, \alpha\rangle}{\langle\alpha, \alpha\rangle}\right), \quad \lambda \in \mathscr{U}_{C}^{*}
$$

Then the map $\lambda \mapsto d_{s}^{-1}(\lambda) \Psi_{\lambda, s}^{\prime}$ extends from the tube $\{\lambda \mid \operatorname{Re}\langle i \lambda, \alpha\rangle>0$ for all $\left.\alpha \in \Sigma^{+} \cap s^{-1} \Sigma^{-}\right\}$to a distribution valued holomorphic function $\Psi_{\lambda, s}$ on $\mathcal{U}_{C}^{*}$. For each $\lambda, \Psi_{\lambda, s}$ is a conical distribution in $D_{\lambda}^{\prime}$.

Assume, in the sequel, that the rank of $X$ equals 1 . Let $s \in W$ be the nontrivial element and $e \in W$ the identity. Let $\alpha$ be the element in $\Sigma_{0}^{+}$, and $m_{\alpha}$ the multiplicity of $\alpha$. Let $d \xi$ be the $G$-invariant measure on $\Xi$.

It is noted in [1] that if $\lambda=0$, all the $\Psi_{\lambda, s}, s \in W$, constructed in Theorem 2 are proportional. The distribution $\Psi_{0}$ in Theorem 3 provides a compensation for this.

TheOREM 3. For $\phi \in D(\Xi)$, let $\phi_{0} \in D(\Xi)$ be given by $\phi_{0}(k a M N)=$ $\phi(a M N)$. Then

$$
\Psi_{0}: \phi \mapsto \int_{\Xi}\left(\phi(\xi)-\phi_{0}(\xi)\right) e^{\rho(\log a(\xi))} d \xi
$$

is a conical distribution in $D_{0}^{\prime}$.

From the construction we see easily that $\Psi_{\lambda, e}$ is concentrated on $\Xi_{e}=$ $A M N$. So is $\Psi_{\lambda, s}$ if $-i \lambda$ is a positive integral multiple of $\alpha$. Conversely, we have

TheOREM 4. Assume $m_{\alpha} \neq 1$. If the conical distribution $\Psi \in D_{\lambda}^{\prime}$ is 
concentrated on $\Xi_{e}$ and not proportional to $\Psi_{\lambda, e}$, then $-i \lambda$ is a positive integral multiple of $\alpha$, and $\Psi$ is a linear combination of $\Psi_{\lambda, e}$ and $\Psi_{\lambda, s}$. (For $G=S O_{0}(1, n), n>2, c f$. [1, Chapter III, Theorem 4.10].)

With some more technical lemmas and the aid of Theorem 4.9 in [1, Chapter III], we finally arrive at the following main result:

THEOREM 5. Assume the symmetric space $G / K$ has rank 1 and dimension $>2$. Let $\Psi \in D_{\lambda}^{\prime}$ be conical. We have

(i) if $\lambda=0$, then $\Psi=c \Psi_{0}+c^{\prime} \Psi_{\lambda, e}$;

(ii) if $\lambda \neq 0$, then $\Psi=c \Psi_{\lambda, s}+c^{\prime} \Psi_{\lambda, e}$, where $c$ and $c^{\prime}$ are constants.

In case the dimension of $G / K=2$, there is one more base element for the conical distributions in $D_{\lambda}^{\prime}$ if $i \lambda=(1 / 2-l) \alpha, l$ being a positive integer. This discrepancy disappears, however, if we modify the definition of conical distributions so that $G$ is the whole (not necessarily connected) isometry group. After this modification, Theorem 5 is valid for all rank one spaces. In this sense, Helgason's conjecture is true for all rank one symmetric spaces.

\section{REFERENCES}

1. S. Helgason, $A$ duality for symmetric spaces with applications to group representations, Advances in Math. 5 (1970), 1-154. MR 41 \#8587.

DEPARTMENT OF MATHEMATICS, NATIONAL TSING HUA UNIVERSITY, HSINCHU, TAIWAN 